University of Nebraska - Lincoln

DigitalCommons@University of Nebraska - Lincoln

$5-2021$

\title{
Component Causes of Infectious Bovine Keratoconjunctivitis - The Role of Moraxella Species in the Epidemiology of Infectious Bovine Keratoconjunctivitis
}

John Dustin Loy

Matthew Hille

Gabriele Maier

Michael L. Clawson

Follow this and additional works at: https://digitalcommons.unl.edu/vetscipapers

Part of the Biochemistry, Biophysics, and Structural Biology Commons, Cell and Developmental Biology Commons, Immunology and Infectious Disease Commons, Medical Sciences Commons, Veterinary Microbiology and Immunobiology Commons, and the Veterinary Pathology and Pathobiology Commons

This Article is brought to you for free and open access by the Veterinary and Biomedical Sciences, Department of at DigitalCommons@University of Nebraska - Lincoln. It has been accepted for inclusion in Papers in Veterinary and Biomedical Science by an authorized administrator of DigitalCommons@University of Nebraska - Lincoln. 


\title{
Component Causes of Infectious Bovine
}

\section{Keratoconjunctivitis - The Role of Moraxella Species in the Epidemiology of Infectious Bovine Keratoconjunctivitis}

\author{
John Dustin Loy, DVM, PhD, DACVM ${ }^{a, *}$, Matthew Hille, DVM ${ }^{a}$, \\ Gabriele Maier, DVM, MPVM, PhD, DACVPM ${ }^{b}$, Michael L. Clawson, PhD ${ }^{c}$
}

\section{KEYWORDS}

- Infectious bovine keratoconjunctivitis - Moraxella bovis - Moraxella bovoculi

- Moraxella ovis • MALDI-TOF MS • Genomics • Pathogenesis

\author{
KEY POINTS \\ - Moraxella bovis can cause infectious bovine keratoconjunctivitis (IBK). \\ - The role of $M$ bovoculi in IBK is not fully understood. \\ - $M$ bovis and $M$ bovoculi appear to undergo genetic recombination with each other or other \\ members of the Moraxellaceae. \\ - Recombination complicates their classification and potential role(s) in IBK pathogenesis. \\ - MALDI-TOF MS is used to identify $M$ bovis, 2 major strains or genotypes of $M$ bovoculi, $M$ \\ ovis, and other members of the Moraxellaceae. \\ - Classification and determination of pathogenesis potential within Moraxella species may \\ be better understood through whole genome sequencing.
}

\section{INTRODUCTION}

Bacterial pathogens have been associated with infectious bovine keratoconjunctivitis (IBK) or pinkeye, from some of the earliest descriptions of the disease. While investigating outbreaks of keratitis contagiosia in Nebraska cattle, one of the first

\footnotetext{
${ }^{a}$ Nebraska Veterinary Diagnostic Center, School of Veterinary Medicine and Biomedical Sciences, University of Nebraska-Lincoln, 4040 East Campus Loop North 115Q NVDC, Lincoln, NE 68583-0907, USA; ${ }^{\text {b }}$ Department of Population Health \& Reproduction, School of Veterinary Medicine, University of California Davis, 1 Shields Avenue, VM3B, Davis, CA 95616, USA; ${ }^{\circ}$ US Meat Animal Research Center, USDA Agriculture Research Service, Clay Center, 844 Road 313, Clay Center, NE 68933, USA

* Corresponding author.

E-mail address: jdloy@unl.edu
} 
documented reports of IBK, Billings described dense clusters of coccoid bacteria in corneal lesions. The organisms were subsequently isolated in pure culture; however, he was unsuccessful in reproducing the disease in healthy animals. ${ }^{1}$ These early observations and experiments highlight some of the discoveries, knowledge gaps, and controversy that remain after 120 years of research on the causality of IBK. This article will focus on bacterial causes of IBK, specifically members of the genus Moraxella, likely the same organisms Billings observed in 1898.

Moraxella bovis ( $M$ bovis) is the most common bacterial species associated with the disease and appears well suited to cause IBK. $M$ bovis possesses numerous adherence and colonization factors, secretes toxins and enzymes that damage bovine cells, and utilizes various mechanisms to avoid immune recognition and enable persistence in hosts. $M$ bovis is the only pathogen to have reproduced IBK-like ocular lesions in various experimental models, including in gnotobiotic calves, indicating that it is capable of inducing disease in the absence of other potential pathogens and with cell-free supernatants, which is evidence for the role of secreted exotoxins in the pathogenicity of IBK. ${ }^{2-4}$ For other members of the genus, primarily Moraxella bovoculi ( $M$ bovoculi), direct causality appears less clear, as reproduction of the disease following experimental infection with pure culture has not yet been achieved. ${ }^{5}$ However, the presence of virulence factors in $M$ bovoculi similar in structure and function to $M$ bovis, along with recent genomic data showing recombination between the 2 species, highlights the complexity of IBK pathogenesis and possible interactions. The role these pathogens play in causing and/or contributing to IBK is reviewed, including virulence factors, studies on causality, recent findings using genomics and mass spectrometry, and detection and differentiation of these organisms in clinical samples.

\section{The Genus Moraxella}

The genus Moraxella represents the type genus within the larger family Moraxellaceae. ${ }^{6}$ Moraxella are gram-negative rods or cocci, which are oxidase and usually catalase positive. ${ }^{7}$ There are 22 validly published species, most of which are parasites or commensals of mucous membranes of mammals. ${ }^{7,8}$ Moraxella is among the most abundant organisms in the upper respiratory and ocular microbiome, and overall abundance of this genus was not found to be associated with risk of IBK in 1 study. 9,10 Three species within the genus have been studied in relation to IBK, and include $M$ bovis, $M$ bovoculi, and Moraxella (formerly Branhamella and Neisseria) ovis ( $M$ ovis). There is likely taxonomic overlap between $M$ ovis and $M$ bovoculi. In the authors' view, $M$ ovis is rare and mostly absent from ocular cultures of cattle, and reports or descriptions of $M$ ovis from the eyes of cattle prior to the recognition of $M$ bovoculi are likely $M$ bovoculi. Both species can appear identical if utilizing only biochemical testing. ${ }^{11}$ This view agrees with several large prevalence or descriptive epidemiology studies that found low numbers or no $M$ ovis in cattle ocular cultures that have occurred since the description of $M$ bovoculi as a separate species using polymerase chain reaction (PCR) or other nonbiochemical methods for identification. ${ }^{12-15}$

\section{MORAXELLA BOVIS- VIRULENCE FACTORS}

Repeats in Toxin

Exotoxins are a hallmark of many bacterial pathogens and repeats-in-toxin (RTX) type toxins found in numerous pathogenic bacteria. RTX toxins are thought to have originated in members of the Pasteurellaceae, and subsequently disseminated among other bacteria through horizontal gene transfer. ${ }^{16} \mathrm{RTX}$ toxins are large, pore-forming proteins with a common structure of glycine and aspartate-rich repeats, and are 
secreted via a type I secretion system, a transporter necessary to enable secretion of toxin from the cytoplasm to the environment. ${ }^{17}$ RTX toxins include Escherichia coli alpha hemolysin, Mannheimia haemolytica leukotoxin, and the Apx family of Actinobacillus pleuropneumoniae toxins. ${ }^{18,19} \mathrm{M}$ bovis RTX toxin, also called hemolysin, cytolysin, or cytotoxin, reacts with monoclonal antibodies to other RTX toxins and forms pores in bovine erythrocytes, demonstrating similarity in structure and function to other RTX toxins. ${ }^{20,21} \mathrm{M}$ bovis RTX toxin is toxic to bovine neutrophils and corneal epithelium, but not human neutrophils. ${ }^{22,23}$ The host cell receptor for $M$ bovis cytotoxin has not been definitively determined, but other closely related toxins bind to $B_{2}$ integrins on leukocytes, and some, like $M$ haemolytica leukotoxin, bind to a host-specific CD18 signal peptide region. ${ }^{18,24,25}$ This property makes the toxin highly specific to certain cell types, which likely leads to clinical signs observed in associated diseases. For example, $M$ bovis RTX toxin is a necessary and sufficient virulence factor for IBK, where toxin-rich supernatants from hemolytic strains have been shown to reproduce IBK-like lesions in vivo, and nonhemolytic strains and supernatants are avirulent or nontoxic. ${ }^{26,27}$ The RTX toxin is expressed in some $M$ bovis strains in part by a gene $(m b x A)$, which is absent in nonhemolytic strains and under the control of an operon, which forms a pathogenicity island. ${ }^{28-30}$ Activity of the toxin is neutralized by rabbit antiserum raised against the carboxy terminus of $m b x A \cdot{ }^{28}$ In addition to $m b x A$, the $\mathrm{RTX}$ operon genes include $m b x \mathrm{C}$ (toxin activation protein), $m b x \mathrm{~B}$ (transport protein), and $m b x \mathrm{D}$ (transport protein) and a flanking protein related to TolC, a secretion protein similar to other RTX toxin operons (type 1 secretion system). ${ }^{17,29} \mathrm{~A}$ high degree of conservation in mbxA has been observed among isolates from diverse geographic origins, which makes this a potential vaccine antigen target. ${ }^{31,32}$

\section{MORAXELLA BOVIS PILI}

Type IV pili, also known as fimbriae or attachment pili, are small structural protein fibers made up of pilin proteins that serve numerous functions. ${ }^{33}$ In $M$ bovis, they facilitate attachment to corneal epithelium, a process that is inhibited by pili-specific antiserum. Pili are a necessary virulence factor, and strains lacking pili are unable to adhere and therefore cannot cause disease. ${ }^{34-36}$ Inhibition of adherence by pili-specific antiserum is serogroup specific, with at least 7 different serogroups described. ${ }^{37}$ Protection from IBK using pili-based vaccines was shown to be serotype specific in 1 study. ${ }^{38}$ Selection and maintenance of pili in culture through selection and passage of agarcorroding colonies with pili enabled successful challenge and colonization models, as pili expression appears to decrease upon passage (Fig. 1). ${ }^{39-41}$ The isolation frequency of piliated colony types from cattle changes based on seasonality and has been observed to positively correlate with levels of UV radiation. ${ }^{42} M$ bovis also expresses 2 mutually exclusive forms of pilin, called Q (quick) and I (intermediate), in addition to serotype level variation conferred by pili.. ${ }^{43,44}$ The expression of pili is reversible and under the control of an inversion region under a single promoter. ${ }^{44,45}$ The $Q$ pili form has been shown to be more effective at binding to corneal epithelium than both I pili forms and non-piliated strains. ${ }^{46} \mathrm{M}$ bovis selectively attaches, presumably through pili, to dark cells in the cornea, those that are older and devoid of membrane ridges called microplicae, when viewed by scanning electron microscopy; the number of these cells is increased by UV light. ${ }^{47}$ This observation has led to the hypothesis that other environmental factors or coinfections that increase the proportion of dark cells in the cornea may potentially enhance the ability of $M$ bovis to colonize. ${ }^{4,48,49}$ Pili are also involved in the formation of biofilms on both biotic and abiotic surfaces; this formation of biofilms seems to confer greater resistance to lysozyme. ${ }^{50,51}$ 

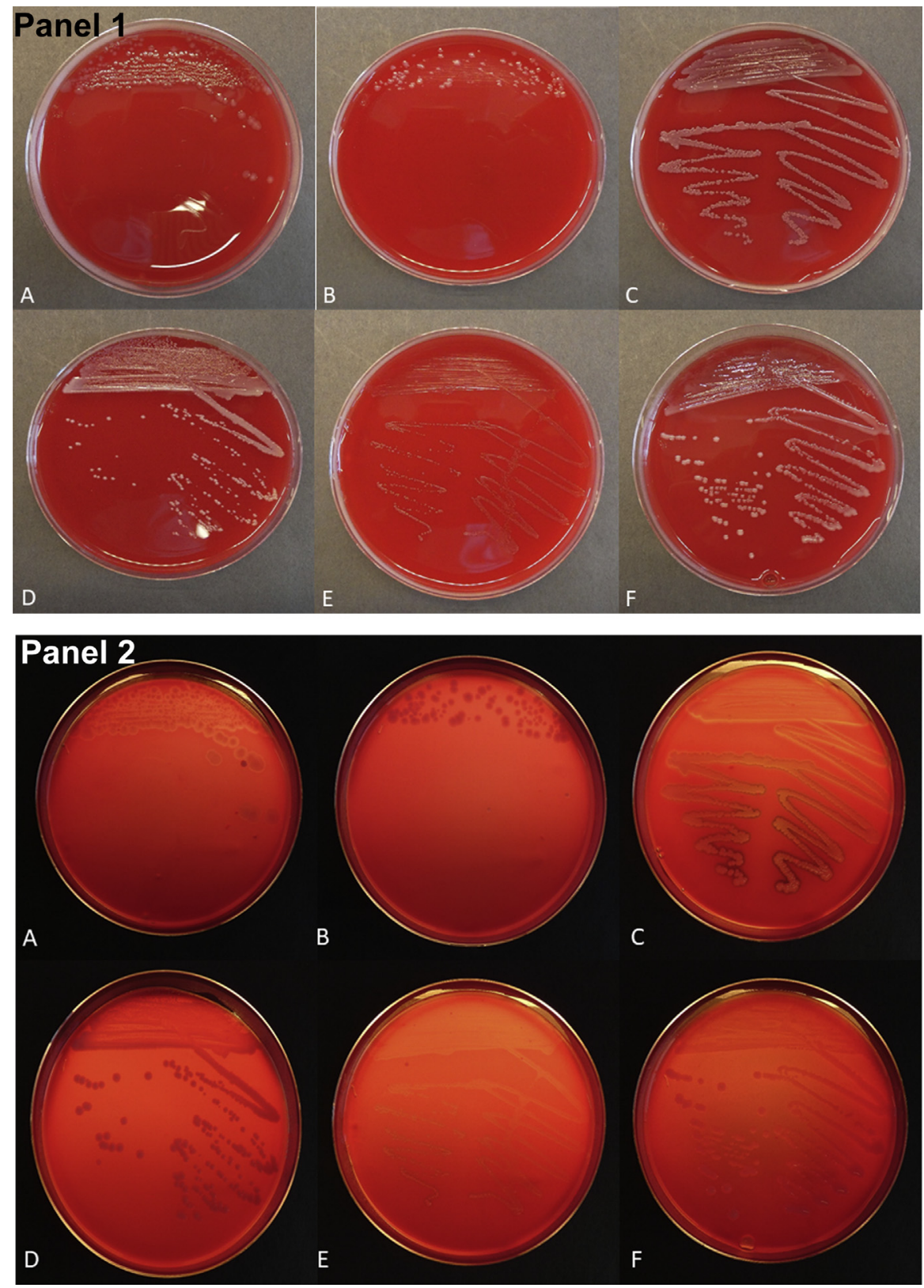

Fig. 1. Panel 1. Differences in Moraxella colony phenotypes. Images were captured after 48hour growth in $5 \% \mathrm{CO}_{2}$ atmosphere on trypticase soy agar with $5 \%$ defibrinated bovine erythrocytes. (A) Strain Epp 63, $M$ bovis, RTX +, beta hemolysis. $(B)$ Isolate 0131, $M$ bovis recombinant, RTX +, beta hemolysis. (C) Isolate Mb58081, M bovoculi, genotype 1, RTX +, beta hemolysis. (D) Isolate Mb58090, M bovoculi, genotype 1, RTX -, gamma hemolysis. (E) Mb58000, M bovoculi, genotype 1, RTX +, stop codon at codon 70 of PilA gene (no pili production), beta hemolysis. ( $F$ ) Mb 68,535, M bovoculi, genotype 2, RTX -, gamma hemolysis. Panel 2. Same plates as panel 1 with backlighting to visualize hemolysis. (Images by Matthew Hille and Justin Lowery.) 


\section{MORAXELLA BOVIS OTHER POTENTIAL VIRULENCE FACTORS}

$M$ bovis possesses an outer membrane (OM) that consists of at least 3 distinct types of lipooligosaccharide (LOS), which are uniquely devoid of heptose. ${ }^{52,53}$ The biologic role of LOS for M bovis was demonstrated, as mutants with truncated LOS (shortened and less functional) had increased susceptibility to the bactericidal activity of bovine serum, decreased adherence to mammalian cells, and increased sensitivity to detergents and some antibiotics. ${ }^{54}$ Some $M$ bovis strains also express a capsular polysaccharide. ${ }^{54,55} \mathrm{OM}$ proteins are the first line of defense against host immune defenses, and these studies provide supportive evidence that in $M$ bovis they may serve as virulence factors.

$M$ bovis strains produce an array of hydrolytic enzymes, which facilitate the breakdown of host protein, lipids, nucleic acids, carbohydrate and fat molecules into their simplest units, and therefore can cause damage to ocular tissues. These include esterases, lipases, phosphoamidase, phosphatase, and hyaluronidase. $M$ bovis strains can also hydrolyze casein, produce fibrinolysin, and are agarolytic. ${ }^{56}$ Some strains express and secrete phospholipase, which is conserved and has been considered by some as a potential vaccine antigen. ${ }^{57}$ Other strains carry a plasmid encoding filamentous haemagglutinin-like proteins ( $f / p A$ and flpB), which may facilitate host colonization, similar to virulence factors found in Bordetella pertussis. ${ }^{58}$ Iron acquisition is a critical function for pathogens to replicate and maintain infections, as iron is tightly regulated in the host environment. Some $M$ bovis strains also possess ironrepressible outer membrane proteins similar to the human pathogen $N$ meningitidis $(\operatorname{IrpA})^{59}$ and have robust iron acquisition machinery, including fur homologues, and are capable of acquiring iron from bovine lactoferrin (lbp) and transferrin (tbp). ${ }^{60,61}$

\section{STUDIES ON MORAXELLA BOVIS AND CAUSALITY}

Experimental studies have definitively demonstrated that $M$ bovis can cause IBK-like lesions compared with uninfected controls. ${ }^{5,62-64}$ However, insults to the cornea such as ultraviolet (UV) radiation or corneal scarification may be necessary in experimental infection studies to induce IBK. ${ }^{5,65}$ Lesions have also been produced in the absence of corneal insults, and whether they are required may depend on the infectious dose or the strain virulence and immune status of the host. ${ }^{63}$ Experimental infections with some strains appear to be enhanced by coinoculation with $M$ bovoculi. ${ }^{66}$ Evidence for the causal role of $M$ bovis in IBK came through experimental infections with strains of known virulence in gnotobiotic calf models, which have reproduced clinical and lesions consistent with IBK, including histopathological changes in the cornea and conjunctiva in the absence of other factors such as trauma or coinfections with other bacterial species. ${ }^{3,4,67}$ Purified fractions of $M$ bovis cell-free supernatants were shown to reproduce IBK lesions in calves, implying a role for a specific excreted $M$ bovis virulence factor to cause IBK, which we now understand to be the RTX toxin encoded by mbxA. ${ }^{2}$

In field studies, $M$ bovis is often isolated from IBK-affected cattle, but it is also found in normal healthy eyes, with variations in isolation rates observed depending on seasonality and animal age. ${ }^{42,68,69}$ One study showed high prevalence of isolation of $M$ bovis in the absence of other agents in outbreaks that were diverse in space and time, but did not include cultures from nondiseased eyes for comparison. ${ }^{70}$ A study of an outbreak during winter, winter pinkeye, had a high prevalence of $M$ bovis isolated in the absence of other agents, and the isolates were able to reproduce IBK in calves, but this study also did not culture healthy eyes for comparison. ${ }^{71}$ More recent studies using PCR methods showed only a weak temporal association between $M$ bovis 
detection and IBK in naturally occurring outbreaks of IBK. ${ }^{14}$ The observation that $M$ bovis is isolated from nonclinical animals at similar rates as those from IBK cases has been documented numerous times in epidemiologic studies, thus complicating the picture of a single infectious agent that is alone necessary and sufficient for causality. ${ }^{72,73}$

The mechanism for why discrepancies between the presence of $M$ bovis and clinical cases of IBK are observed could be comparable to what is understood about bovine respiratory disease (BRD). Many of the pathogens associated with BRD can be isolated from the respiratory tract of healthy animals. Immunomodulatory effects of common bovine viral infections or stress allow for these opportunistic pathogens to replicate, invade deeper into the respiratory tract, secrete toxins, and cause disease. ${ }^{74}$ Likewise, $M$ bovis may only be able to cause IBK if other conditions are met, such as stress, physical damage, UV radiation, or face fly irritation. Also, with BRD there are well-defined strains, or genotypes, of $M$ haemolytica that differ by their armament of virulence factors and outer membrane proteins, and by their association with BRD. ${ }^{75,76}$ The same could be true for $M$ bovis and IBK, because of changes in alleles throughout the genome that alter or enhance virulence. More detailed discussion about causal models for IBK is available elsewhere in this issue.

\section{MORAXELLA BOVIS GENOMICS}

Genetic diversity among $M$ bovis has been examined through DNA fingerprinting with enterobacterial repetitive intergenic consensus (ERIC), and random amplified polymorphic DNA (RAPD). ${ }^{53,77}$ Recently, the first complete whole-genome sequence of the Epp63 strain, used in many studies, was published. ${ }^{78}$ Aside from having known virulence factors including an RTX operon, multiple pilin and prepilin genes, and other genes involved with adhesion, the Epp63 genome has numerous repetitive regions. These include 2 repeat regions associated with CRISPR and 5800 repeat regions consisting of dinucleotide to decanucleotide units, ranging in size from 8 to 422 bases. The repeats are located throughout the genome, either in noncoding regions, coding regions, or both, which suggests that $M$ bovis may employ slipped strand mispairing coupled to phase variation as a way to adapt to particular niche environments including changing host immune responses directed at select $M$ bovis antigens. Multiple pathogens employ this technique, which may enable $M$ bovis strains to vary their pathogenicity by the phase state of their genomes. ${ }^{79}$ On a practical level, multiple repeat regions probably explain why there is a paucity of fully assembled $M$ bovis genomes in public databases at present, as short reads of a sequence commonly produced by most sequencing technologies would not span these large repeat regions, which would interfere with the assembly of a complete $M$ bovis genome. Additional complete genomes will be required to understand $M$ bovis genomic diversity better, as well as strain pathogenicity or lack thereof, and the extent of recombination between $M$ bovis and other members of the Moraxellaceae.

\section{MORAXELLA BOVOCULI Virulence Factors}

Several virulence factors homologous to those found in $M$ bovis have been found within $M$ bovoculi, which provides partial support for the hypothesis that it may play a role in IBK pathogenesis, despite not having been able to replicate IBK-like lesions in experimental infections. The 2 most significant homologous virulence factors are a complete RTX operon and type IV pilin proteins. ${ }^{80,81}$ The mbvA gene, responsible for the RTX toxin (also called cytotoxin A) within $M$ bovoculi is highly conserved with 
99.3\% nucleotide and $98.8 \%$ corresponding amino acid homology within the species. $^{32}$ Similar to $M$ bovis, the RTX toxin of $M$ bovoculi is necessary for hemolytic activity (Fig. 2). ${ }^{80,82}$ Although the RTX toxins produced by $M$ bovis and $M$ bovoculi are similar in structure and function, within an in vitro model using bovine erythrocytes, polyclonal serum raised against $M$ bovoculi $R T X$ toxin did not neutralize corresponding $M$ bovis toxin activity encoded by $m b x A$, but did neutralize $M$ ovis RTX toxin, thus indicating sufficient differences between $M$ bovis and $M$ bovoculi toxins in structure to require additional immune responses. ${ }^{80}$ Some of these RTX-related operon sequences found in $M$ bovoculi demonstrate what appears to be interspecies mosaicism, where recombination with $M$ bovis RTX-related sequences ( $m b v B$ and $m b v D$ ) is apparent in some sequences. ${ }^{83}$

Sequence analysis of $M$ bovoculi pilin (pilA) appears to be substantially different than that of $M$ bovis, with as little as $38 \%$ sequence homology between the 2 species. ${ }^{81}$ However, $M$ bovoculi pilin sequences show limited intraspecies diversity in contrast to $M$ bovis. ${ }^{83,84}$ The large diversity between species suggests that any cross-protection to pilin epitopes would be minimal. The potential importance of the few variable regions within $M$ bovoculi pilin sequences is unknown. If these variable regions prove to be important clinically, it could explain the failures thus far to produce disease experimentally and/or provide an effective immune response using a limited number of isolates, as the variable regions may be important determinants of initial ocular colonization and disease initiation, as observed with $M$ bovis.

Like $M$ bovis, $M$ bovoculi has also been shown to form biofilms on abiotic surfaces such as polystyrene microplates. ${ }^{51}$ Biofilms have a well-established association with disease severity in numerous microbial diseases of people and animals. ${ }^{85,86}$ Biofilm formation is variable within $M$ bovoculi, and also could potentially contribute to variation in virulence. For example, the type strain of $M$ bovoculi (BAA1259) has moderate biofilm-forming capacity, where more recently isolated field strains of $M$ bovoculi used in the study had stronger biofilm-forming capability. ${ }^{51}$ Given the relatedness of the species, the reliance on type IV pilus for biofilm formation would seem likely, but this has not been examined within $M$ bovoculi.

\section{CHALLENGE MODELS}

In 1 study, $M$ bovoculi did not cause IBK in conventionally reared calves, even though a hemolytic isolate representing the type strain was used for the challenge. In this same study, controls using $M$ bovis strain Epp63 did cause disease consistent with

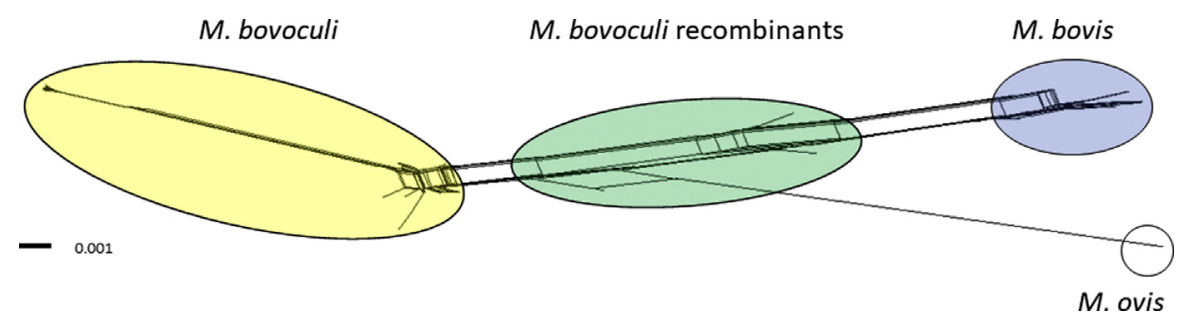

Fig. 2. Neighbor-joining network made in SplitsTree of full length $16 \mathrm{~S}$ sequences. The network contains $18 \mathrm{M}$ bovis sequences (contained in the blue ellipse and described by Robbins and colleagues), $253 \mathrm{M}$ bovoculi non-recombinant and recombinant sequences (contained in the yellow and green ellipses, respectively, and described by Dickey and colleagues, GenBank\# SRP070887) and $2 M$ ovis sequences (contained in the white circle, GenBank \# AF005186 and NR_028670). The scale bar equals substitutions per site. 
IBK using the same randomized and blinded challenge model. ${ }^{5}$ These findings are in agreement with a smaller study from 25 years prior in which $M$ ovis (likely $M$ bovoculi) did not cause corneal lesions in a small group of calves. ${ }^{87}$ Gould and colleagues ${ }^{60}$ recognized this older study was inadequate because of a lack of a positive control, meaning it was not possible to determine if the organism did not cause disease or the model was incapable of causing disease. Regardless, $M$ bovoculi has not demonstrated a causal relationship to IBK under experimental conditions. However, genomic diversity within $M$ bovoculi further complicates interpreting this challenge work, as only limited strains have been used for experimental inoculations. ${ }^{83,88}$

\section{MORAXELLA BOVOCULI AND CAUSALITY}

Despite not having shown to reproduce the disease experimentally, a hallmark of infectious disease causation in veterinary science, there are some findings that support a potential contributive or associative role for $M$ bovoculi in IBK pathogenesis. Prior to the characterization of $M$ bovoculi, isolates classified as $M$ ovis (likely $M$ bovoculi) were isolated three times more often than $M$ bovis from clinical samples of IBK cases submitted to a diagnostic laboratory. ${ }^{82}$ More recently, $M$ bovoculi was the only bacteria isolated from most (64\%) of individual animal cases of IBK over a 4-year timeframe with only $22 \%$ of cases isolating only $M$ bovis. ${ }^{13}$ In this same study, the authors found when examining cases where more than 1 bacterial species was isolated, $M$ bovoculi and $M$ bovis were isolated from $74 \%$ and $32 \%$ of cases, respectively. ${ }^{13}$ Another study utilizing real-time PCR detection on diagnostic laboratory submissions found similar results, where $75.9 \%$ of samples (136/179) had $M$ bovoculi detected. ${ }^{89}$ In studies looking at prevalence of Moraxella, one study showed that only the detection of $M$ bovoculi by PCR methods was associated with clinical signs of IBK. This same study also showed that prevalence of $M$ bovoculi may be associated with likelihood of undergoing an acute IBK outbreak. ${ }^{68}$ Another study showed the recovery of $M$ bovoculi and $M$ bovis was more frequent from eyes with IBK lesions than unaffected eyes. ${ }^{14}$ It also showed that calves infected with $M$ bovis had a higher subsequent risk of developing IBK than calves infected with $M$ bovoculi. ${ }^{14}$ Caution should be taken to avoid overinterpretation of postlesion microbiological data, which does not establish temporality between presence of pathogens and occurrence of lesions. ${ }^{5}$ Documenting that exposure occurs before disease is essential for establishing causation in future studies.

\section{MORAXELLA BOVOCULI GENOMICS}

$M$ bovoculi has recently been classified into 2 major strain types, or genotypes ( 1 and 2 ), based on whole-genome sequencing of over 200 isolates. ${ }^{83,88}$ The genotypes differ in several ways. Both genotype 1 and $2 \mathrm{M}$ bovoculi have been isolated from the eyes of cattle without IBK, while only genotype $1 \mathrm{M}$ bovoculi, which includes the type strain, has been isolated and identified from the eyes of cattle with IBK. However, the frequency and potential role, or lack thereof, of genotype $2 M$ bovoculi in IBK remain poorly understood. Genotype $2 M$ bovoculi has only recently been described, and isolates may have been inadvertently misclassified as non-bovoculi Moraxella in the past based on a PCR typing method instead of biochemical for $M$ bovoculi that was developed prior to their discovery. ${ }^{88,90}$ The PCR-based typing method only identifies genotype 1 strains, as genotype 2 strains do not contain the restriction enzyme binding site targeted in the method to distinguish $M$ bovoculi from $M$ ovis and other Moraxella species. ${ }^{83,90,91}$ Thus, the difference between the genotypes in IBK-positive or -negative eyes needs to be tested in more studies that do not have biases in isolate 
identification. Recently, a MALDI-TOF biomarker method, which uses mass spectrometry peaks that are genotype specific to classify $M$ bovoculi isolates, was developed that would make these data easier to generate, as these instruments are now available in many diagnostic laboratories. ${ }^{92}$

Genotype $1 \mathrm{M}$ bovoculi appears to be more diverse than genotype 2. Over 127,000 SNPs have been identified within and across the $2 M$ bovoculi genotypes, with over $80 \%$ characterizing diversity exclusively in genotype 1 strains. ${ }^{83}$ The 2 genotypes also appear to differ in their RTX profiles. To date, no genotype 2 strains have been found to have an RTX operon versus $85 \%$ of genotype 1 strains isolated from IBK eyes that do, including the type strain. ${ }^{83}$ Thus, current studies suggest that $M$ bovoculi genotypes may differ in their propensity to cause or contribute to the development of IBK, with genotype 1 isolates more likely to have RTX, and extensive genetic diversity, and genotype 2 isolates having genomic profiles more consistent with commensals.

\section{CLASSIFICATION OF MORAXELLA BOVIS AND MORAXELLA BOVOCULI}

MALDI-TOF assays that enable identification of $M$ bovis and the 2 genotypes of $M$ bovoculi may be improved or enhanced as more is learned about the 2 species and the extent of recombination that may have taken place between them. There is compelling evidence that $M$ bovoculi has recombinant $16 S$ rDNA alleles (see Fig. 2) that were possibly acquired from $M$ bovis, and $M$ bovis has recombinant alleles within the internal transcribed spacer region of the ribosomal locus. ${ }^{91}$ Given the high conservation of the ribosomal locus throughout the evolution of bacteria, it is not unexpected that these species have extensive recombination elsewhere throughout their genomes. ${ }^{83}$ This observation challenges assignment of IBK causality, as these mixed chimeric genomes may not be easily assigned to 1 species. However, it is possible to distinguish $M$ bovis, $M$ bovoculi, and $M$ bovoculi recombinants from each other with full-length $16 \mathrm{~S}$ rDNA sequences (see Fig. 2). Full-genome sequences will ultimately be much more powerful in classifying these bacteria as an identity -by -state combination of alleles acquired through vertical descent and horizontal lateral gene transfer events. The same may be true for other members of the Moraxellaceae, and methods that classify members of this family using full, or particularly partial $16 \mathrm{~S}$ sequences, may be shown to conflate members of the family. Examples include $M$ ovis and members of the closely related genus $P$ sychrobacter, which are also found in cattle and have been isolated from bovine eye swabs by the authors. ${ }^{93,94}$

\section{SUMMARY AND FUTURE WORK}

Whole-genome sequencing of $M$ bovis and $M$ bovoculi strains isolated from the eyes of cattle with or without IBK, followed by genome comparisons, may reveal core genetic determinants that are necessary for virulence and further understanding of the diversity and contributions of these organisms to IBK.

\section{CLINICS CARE POINTS- DIAGNOSIS AND ESTABLISHMENT OF MORAXELLA ETIOLOGY IN INFECTIOUS BOVINE KERATOCONJUNCTIVITIS}

- Determining the etiology in individual outbreaks is challenging, because Moraxella species have been isolated from cases of IBK and normal eyes, and the isolation of an agent after the appearance of a lesion does not establish causation.

- For microbiological sample collection, the ocular conjunctival sac is aggressively swabbed to culture for pathogens while avoiding contamination. Use of flocked 
swabs in liquid transport media offers enhanced collection and release of bacteria into the media. ${ }^{95}$

- False-negative culture results can occur if there is contamination or multiple pathogens.

- PCR as a culture independent modality can be useful, although caveats and challenges related to classification and misclassification of Moraxella, as discussed, also apply to PCR diagnostic methods.

- A multiplexed real-time PCR is available that targets the toxin genes for $M$ bovis ( $m b x A)$ and $M$ bovoculi $(m b v A) .{ }^{89}$ Similar approaches for bovine respiratory disease pathogens have been shown to increase co-detections over culture alone. ${ }^{96}$

- The authors routinely see case submissions from field outbreaks of IBK where only $M$ bovoculi is isolated by culture, but similar levels (Ct values) of $M$ bovis toxin genes are detected by real-time PCR, which indicates the potential for false-negative $M$ bovis cultures.

- Newly developed MALDI-TOF assays provide quick, accurate, and economic methods to identify Moraxella species from culture. These methods also allow differentiation of $M$ bovoculi isolates traditionally associated with disease versus those that likely represent normal flora. ${ }^{91,92}$

\section{DISCLOSURE}

The authors have nothing to disclose.

\section{REFERENCES}

1. Billings FS. Keratitis contagiosa in cattle. Nebr Agric Exp Station Bull 1889;10: 246-52.

2. Beard MK, Moore LJ. Reproduction of bovine keratoconjunctivitis with a purified haemolytic and cytotoxic fraction of Moraxella bovis. Vet Microbiol 1994;42(1): 15-33.

3. Rogers DG, Cheville NF, Pugh GW Jr. Pathogenesis of corneal lesions caused by Moraxella bovis in gnotobiotic calves. Vet Pathol 1987;24(4):287-95.

4. Chandler R, Turfrey B, Smith K, et al. Virulence of Moraxella bovis in gnotobiotic calves. Vet Rec 1980;106(16):364-5.

5. Gould S, Dewell R, Tofflemire K, et al. Randomized blinded challenge study to assess association between Moraxella bovoculi and infectious bovine keratoconjunctivitis in dairy calves. Vet Microbiol 2013;164(1-2):108-15.

6. Rossau R, Van landschoot A, Gillis M, et al. Taxonomy of Moraxellaceae fam. nov., a new bacterial family to accommodate the genera Moraxella, Acinetobacter, and Psychrobacter and related organisms. Int J Syst Evol Microbiol 1991;41(2):310-9.

7. Moraxella. Bergey's manual of systematics of archaea and bacteria. 2012. p. 1-17.

8. Parte AC, Sardà Carbasse J, Meier-Kolthoff JP, et al. List of prokaryotic names with standing in nomenclature (LPSN) moves to the DSMZ. Int J Syst Evol Microbiol 2020;70:5607-12.

9. Cullen JN, Lithio A, Seetharam AS, et al. Microbial community sequencing analysis of the calf eye microbiota and relationship to infectious bovine keratoconjunctivitis. Vet Microbiol 2017;207:267-79.

10. Nicola I, Cerutti F, Grego E, et al. Characterization of the upper and lower respiratory tract microbiota in Piedmontese calves. Microbiome 2017;5(1):152. 
11. Angelos JA. Moraxella bovoculi and infectious bovine keratoconjunctivitis: cause or coincidence? Vet Clin North Am Food Anim Pract 2010;26(1):73-8, table of contents.

12. Maboni G, Gressler LT, Espindola JP, et al. Differences in the antimicrobial susceptibility profiles of Moraxella bovis, M bovoculi and M ovis. Braz J Microbiol 2015;46(2):545-9.

13. Loy JD, Brodersen BW. Moraxella spp. isolated from field outbreaks of infectious bovine keratoconjunctivitis: a retrospective study of case submissions from 2010 to 2013. J Vet Diagn Invest 2014;26(6):761-8.

14. O'Connor AM, Shen HG, Wang C, et al. Descriptive epidemiology of Moraxella bovis, Moraxella bovoculi and Moraxella ovis in beef calves with naturally occurring infectious bovine keratoconjunctivitis (pinkeye). Vet Microbiol 2012;155(2-4): 374-80.

15. Angelos JA, Spinks PQ, Ball LM, et al. Moraxella bovoculi sp. nov., isolated from calves with infectious bovine keratoconjunctivitis. Int J Syst Evol Microbiol 2007; 57(Pt 4):789-95.

16. Frey J, Kuhnert P. RTX toxins in Pasteurellaceae. Int J Med Microbiol 2002;292(3): 149-58.

17. Linhartova I, Osicka R, Bumba L, et al. RTX toxins: a review. In: Gopalakrishnakone P, Stiles B, Alape-Girón A, et al, editors. Microbial toxins. Dordrecht: Springer Netherlands; 2016. p. 1-29.

18. Frey J. RTX toxins of animal pathogens and their role as antigens in vaccines and diagnostics. Toxins (Basel) 2019;11(12):719.

19. Welch RA. Pore-forming cytolysins of gram-negative bacteria. Mol Microbiol $1991 ; 5(3): 521-8$.

20. Gray JT, Fedorka-Cray PJ, Rogers DG. Partial characterization of a Moraxella bovis cytolysin. Vet Microbiol 1995;43(2-3):183-96.

21. Clinkenbeard KD, Thiessen AE. Mechanism of action of Moraxella bovis hemoIysin. Infect Immun 1991;59(3):1148-52.

22. Kagonyera GM, George LW, Munn R. Cytopathic effects of Moraxella bovis on cultured bovine neutrophils and corneal epithelial cells. Am J Vet Res 1989; 50(1):10-7.

23. Kagonyera GM, George L, Miller M. Effects of Moraxella bovis and culture filtrates on 51Cr-labeled bovine neutrophils. Am J Vet Res 1989;50(1):18-21.

24. Shanthalingam S, Srikumaran S. Intact signal peptide of CD18, the beta-subunit of beta2-integrins, renders ruminants susceptible to Mannheimia haemolytica leukotoxin. Proc Natl Acad Sci U S A 2009;106(36):15448-53.

25. Li J, Clinkenbeard KD, Ritchey JW. Bovine CD18 identified as a species specific receptor for Pasteurella haemolytica leukotoxin. Vet Microbiol 1999;67(2):91-7.

26. Beard MK, Moore LJ. Reproduction of bovine keratoconjunctivitis with a purified haemolytic and cytotoxic fraction of Moraxella bovis. Vet Microbiol 1994;42(1): 15-33.

27. Pugh GW Jr, Hughes DE. Experimental bovine infectious keratoconjunctivitis caused by sunlamp irradiation and Moraxella bovis infection: correlation of haemolytic ability and pathogenicity. Am J Vet Res 1968;29(4):835-9.

28. Angelos JA, Hess JF, George LW. Cloning and characterization of a Moraxella bovis cytotoxin gene. Am J Vet Res 2001;62(8):1222-8.

29. Angelos JA, Hess JF, George LW. An RTX operon in hemolytic Moraxella bovis is absent from nonhemolytic strains. Vet Microbiol 2003;92(4):363-77.

30. Hess JF, Angelos JA. The Moraxella bovis RTX toxin locus mbx defines a pathogenicity island. J Med Microbiol 2006;55(4):443-9. 
31. Angelos JA, Ball LM. Relatedness of cytotoxins from geographically diverse isolates of Moraxella bovis. Vet Microbiol 2007;124(3-4):382-6.

32. Farias LDA, Maboni G, Matter LB, et al. Phylogenetic analysis and genetic diversity of $3^{\prime}$ region of rtxA gene from geographically diverse strains of Moraxella bovis, Moraxella bovoculi and Moraxella ovis. Vet Microbiol 2015;178(3):283-7.

33. Giltner CL, Nguyen Y, Burrows LL. Type IV pilin proteins: versatile molecular modules. Microbiol Mol Biol Rev 2012;76(4):740-72.

34. Moore LJ, Rutter JM. Attachment of Moraxella bovis to calf corneal cells and inhibition by antiserum. Aust Vet J 1989;66(2):39-42.

35. Jayappa HG, Lehr C. Pathogenicity and immunogenicity of piliated and nonpiliated phases of Moraxella bovis in calves. Am J Vet Res 1986;47(10):2217-21.

36. Gil-Turnes C. Hemagglutination, autoagglutination and pathogenicity of Moraxella bovis strains. Can J Comp Med 1983;47(4):503-4.

37. Moore LJ, Lepper AW. A unified serotyping scheme for Moraxella bovis. Vet Microbiol 1991;29(1):75-83.

38. Lepper AWD, Moore LJ, Atwell JL, et al. The protective efficacy of pili from different strains of Moraxella bovis within the same serogroup against infectious bovine keratoconjunctivitis. Vet Microbiol 1992;32(2):177-87.

39. Lepper AW. Pink Eye of Cattle. In: Butcher B, editor. Of vets, viruses and vaccines: the story of CSIRO's Animal Health Research Laboratory. Collingwood, Vic: CSIRO; 2000. p. 194-9.

40. Ruehl WW, Marrs CF, Fernandez R, et al. Purification, characterization, and pathogenicity of Moraxella bovis pili. The J Exp Med 1988;168(3):983-1002.

41. Pedersen KB, Frøholm LO, Bøvre K. Fimbriation and colony type of Moraxella bovis in relation to conjunctival colonization and development of keratoconjunctivitis in cattle. Acta Pathol Microbiol Scand B Microbiol Immunol 1973;80B(6):911-8.

42. Lepper AW, Barton IJ. Infectious bovine keratoconjunctivitis: seasonal variation in cultural, biochemical and immunoreactive properties of Moraxella bovis isolated from the eyes of cattle. Aust Vet J 1987;64(2):33-9.

43. Marrs CF, Schoolnik G, Koomey JM, et al. Cloning and sequencing of a Moraxella bovis pilin gene. J Bacteriol 1985;163(1):132-9.

44. Marrs CF, Ruehl WW, Schoolnik GK, et al. Pilin-gene phase variation of Moraxella bovis is caused by an inversion of the pilin genes. J Bacteriol 1988;170(7): 3032-9.

45. Fulks KA, Marrs CF, Stevens SP, et al. Sequence analysis of the inversion region containing the pilin genes of Moraxella bovis. J Bacteriol 1990;172(1):310-6.

46. Ruehl WW, Marrs C, Beard MK, et al. Q pili enhance the attachment of Moraxella bovis to bovine corneas in vitro. Mol Microbiol 1993;7(2):285-8.

47. Vogelweid CM, Miller RB, Berg JN, et al. Scanning electron microscopy of bovine corneas irradiated with sunlamps and challenge exposed with Moraxella bovis. Am J Vet Res 1986;47(2):378-84.

48. Jackman SH, Rosenbusch RF. In vitro adherence of Moraxella bovis to intact corneal epithelium. Curr Eye Res 1984;3(9):1107-12.

49. Chandler RL, Bird RG, Smith MD, et al. Scanning electron microscope studies on preparations of bovine cornea exposed to Moraxella bovis. J Comp Pathol 1983; 93(1):1-8.

50. Prieto C, Serra DO, Martina P, et al. Evaluation of biofilm-forming capacity of Moraxella bovis, the primary causative agent of infectious bovine keratoconjunctivitis. Vet Microbiol 2013;166(3):504-15. 
51. Ely VL, Vargas AC, Costa MM, et al. Moraxella bovis, Moraxella ovis and Moraxella bovoculi: biofilm formation and lysozyme activity. J Appl Microbiol 2019; 126(2):369-76.

52. De Castro C, Grice ID, Daal TM, et al. Elucidation of the structure of the oligosaccharide from wild type Moraxella bovis Epp63 lipooligosaccharide. Carbohydr Res 2014;388:81-6.

53. Prieto Cl, Aguilar OM, Yantorno OM. Analyses of lipopolysaccharides, outer membrane proteins and DNA fingerprints reveal intraspecies diversity in Moraxella bovis isolated in Argentina. Vet Microbiol 1999;70(3-4):213-23.

54. Singh S, Grice ID, Peak IR, et al. The role of lipooligosaccharide in the biological activity of Moraxella bovis strains Epp63, Mb25 and L183/2, and isolation of capsular polysaccharide from L183/2. Carbohydr Res 2018;467:1-7.

55. Wilson JC, Hitchen PG, Frank M, et al. Identification of a capsular polysaccharide from Moraxella bovis. Carbohydr Res 2005;340(4):765-9.

56. Frank SK, Gerber JD. Hydrolytic enzymes of Moraxella bovis. J Clin Microbiol 1981;13(2):269-71.

57. Farn JL, Strugnell RA, Hoyne PA, et al. Molecular characterization of a secreted enzyme with Phospholipase B Activity from Moraxella bovis. J Bacteriol 2001; 183(22):6717-20.

58. Kakuda T, Sarataphan N, Tanaka T, et al. Filamentous-haemagglutinin-like protein genes encoded on a plasmid of Moraxella bovis. Vet Microbiol 2006;118(1): $141-7$.

59. Kakuda T, Oishi D, Tsubaki S, et al. Molecular cloning and characterization of a 79-kDa iron-repressible outer-membrane protein of Moraxella bovis. FEMS Microbiol Lett 2003;225(2):279-84.

60. Kakuda T, Oishi D, Tsubaki S, et al. Cloning and characterization of the fur gene from Moraxella bovis. Microbiol Immunol 2003;47(6):411-7.

61. Yu R-H, Schryvers AB. Bacterial lactoferrin receptors: insights from characterizing the Moraxella bovis receptors. Biochem Cell Biol 2002;80(1):81-90.

62. Aikman JG, Allan EM, Selman IE. Experimental production of infectious bovine keratoconjunctivitis. Vet Rec 1985;117(10):234-9.

63. Chandler RL, Baptista PJ, Turfrey B. Studies on the pathogenicity of Moraxella bovis in relation to infectious bovine keratoconjunctivitis. J Comp Pathol 1979; 89(3):441-8.

64. Henson JB, Grumbles LC. Infectious bovine keratoconjunctivitis. I. Etiology. Am J Vet Res 1960;21:761-6.

65. Weech GM, Renshaw HW. Infectious bovine keratoconjunctivitis: bacteriologic, immunologic, and clinical responses of cattle to experimental exposure with Moraxella bovis. Comp Immunol Microbiol Infect Dis 1983;6(1):81-94.

66. Rosenbusch RF. Influence of mycoplasma preinfection on the expression of Moraxella bovis pathogenicity. Am J Vet Res 1983;44(9):1621-4.

67. Rogers DG, Cheville NF, Pugh GW Jr. Conjunctival lesions caused by Moraxella bovis in gnotobiotic calves. Vet Pathol 1987;24(6):554-9.

68. Schnee C, Heller M, Schubert E, et al. Point prevalence of infection with Mycoplasma bovoculi and Moraxella spp. in cattle at different stages of infectious bovine keratoconjunctivitis. Vet J 2015;203(1):92-6.

69. Bartenslager AC, Althuge ND, Loy JD, et al. Longitudinal assessment of the bovine ocular bacterial community dynamics in calves. Anim Microbiome 2021; 3(1): 16 .

70. Wilcox GE. The aetiology of infectious bovine keratoconjunctivitis in Queensland 1. Moraxella bovis. Aust Vet J 1970;46(9):409-14. 
71. Pugh GW Jr, Hughes DE. Bovine infectious keratoconjunctivitis: Moraxella bovis as the sole etiologic agent in a winter epizootic. J Am Vet Med Assoc 1972; 161(5):481-6.

72. Barber DM, Jones GE, Wood A. Microbial flora of the eyes of cattle. Vet Rec 1986; 118(8):204-6.

73. Pugh GW Jr, McDonald TJ. Identification of bovine carriers of Moraxella bovis by comparative cultural examinations of ocular and nasal secretions. Am J Vet Res 1986;47(11):2343-5.

74. Mosier D. Review of BRD pathogenesis: the old and the new. Anim Health Res Rev 2014;15(2):166-8.

75. Clawson ML, Murray RW, Sweeney MT, et al. Genomic signatures of Mannheimia haemolytica that associate with the lungs of cattle with respiratory disease, an integrative conjugative element, and antibiotic resistance genes. BMC Genomics 2016;17(1):982.

76. Clawson ML, Schuller G, Dickey AM, et al. Differences between predicted outer membrane proteins of genotype 1 and 2 Mannheimia haemolytica. BMC Microbiol 2020;20(1):250.

77. Comin HB, Domingues R, Gaspar EB, et al. Genetic differences among Moraxella bovis and Moraxella bovoculi isolates from infectious bovine keratoconjunctivitis (IBK) outbreaks in southern Brazil. Genet Mol Biol 2020;43(2): e20180380-e20180380.

78. Loy JD, Dickey AM, Clawson ML. Complete genome sequence of Moraxella bovis strain Epp-63 (300), an etiologic agent of infectious bovine keratoconjunctivitis. Microbiol Resour Announc 2018;7(8):e01004-18.

79. van der Woude MW, Baumler AJ. Phase and antigenic variation in bacteria. Clin Microbiol Rev 2004;17(3):581-611, table of contents.

80. Angelos JA, Ball LM, Hess JF. Identification and characterization of complete RTX operons in Moraxella bovoculi and Moraxella ovis. Vet Microbiol 2007; 125(1-2):73-9.

81. Calcutt MJ, Foecking MF, Martin NT, et al. Draft genome sequence of Moraxella bovoculi strain 237T (ATCC BAA-1259T) isolated from a calf with infectious bovine keratoconjunctivitis. Genome Announc 2014;2(3):e00612-4.

82. Cerny HE, Rogers DG, Gray JT, et al. Effects of Moraxella (Branhamella) ovis culture filtrates on bovine erythrocytes, peripheral mononuclear cells, and corneal epithelial cells. J Clin Microbiol 2006;44(3):772-6.

83. Dickey AM, Schuller G, Loy JD, et al. Whole genome sequencing of Moraxella bovoculi reveals high genetic diversity and evidence for interspecies recombination at multiple loci. PLoS One 2018;13(12):e0209113.

84. Angelos JA, Clothier KA, Agulto RL, et al. Relatedness of type IV pilin PilA amongst geographically diverse Moraxella bovoculi isolated from cattle with infectious bovine keratoconjunctivitis. J Med Microbiol 2021;70:001293.

85. Costerton JW, Stewart PS, Greenberg EP. Bacterial biofilms: a common cause of persistent infections. Science 1999;284(5418):1318-22.

86. Abdullahi UF, Igwenagu E, Mu'azu A, et al. Intrigues of biofilm: a perspective in veterinary medicine. Vet World 2016;9(1):12-8.

87. Rosenbusch RF, Ostle AG. Mycoplasma bovoculi infection increases ocular colonization by Moraxella ovis in calves. Am J Vet Res 1986;47(6):1214-6.

88. Dickey AM, Loy JD, Bono JL, et al. Large genomic differences between Moraxella bovoculi isolates acquired from the eyes of cattle with infectious bovine keratoconjunctivitis versus the deep nasopharynx of asymptomatic cattle. Vet Res 2016;47(1):31. 
89. Zheng W, Porter E, Noll L, et al. A multiplex real-time PCR assay for the detection and differentiation of five bovine pinkeye pathogens. J Microbiol Methods 2019; 160:87-92.

90. Angelos JA, Ball LM. Differentiation of Moraxella bovoculi sp. nov. from other coccoid Moraxellae by the use of polymerase chain reaction and restriction endonuclease analysis of amplified DNA. J Vet Diagn Invest 2007;19(5):532-4.

91. Robbins KJ, Dickey AM, Clawson ML, et al. Application and evaluation of the matrix-assisted laser desorption ionization time of flight (MALDI-TOF) mass spectrometry method to identify Moraxella bovoculi and Moraxella bovis isolates from cattle. J Vet Diagn Invest 2018;30(5):739-42.

92. Hille M, Dickey A, Robbins K, et al. Rapid differentiation of Moraxella bovoculi genotypes 1 and 2 using MALDI-TOF mass spectrometry profiles. J Microbiol Methods 2020;173:105942.

93. Holman DB, Timsit E, Amat S, et al. The nasopharyngeal microbiota of beef cattle before and after transport to a feedlot. BMC Microbiol 2017;17(1):70.

94. Shen HG, Gould S, Kinyon J, et al. Development and evaluation of a multiplex real-time PCR assay for the detection and differentiation of Moraxella bovis, Moraxella bovoculi and Moraxella ovis in pure culture isolates and lacrimal swabs collected from conventionally raised cattle. J Appl Microbiol 2011;111(5): 1037-43.

95. Van Horn KG, Audette CD, Tucker KA, et al. Comparison of 3 swab transport systems for direct release and recovery of aerobic and anaerobic bacteria. Diagn Microbiol Infect Dis 2008;62(4):471-3.

96. Loy JD, Leger L, Workman AM, et al. Development of a multiplex real-time PCR assay using two thermocycling platforms for detection of major bacterial pathogens associated with bovine respiratory disease complex from clinical samples. J Vet Diagn Invest 2018;30(6):837-47. 